\title{
Analysis of the determinants of service headway variability at tollbooths under mixed traffic scenario in emerging countries
}

\author{
Yogeshwar V. Navandar ${ }^{1}$, Chintaman Santosh Bari ${ }^{2}$, Ashish Dhamaniya ${ }^{2}$, \\ Shriniwas S. Arkatkar ${ }^{2, *}$ and D. A. Patel ${ }^{2}$ \\ ${ }^{1}$ Department of Civil Engineering, NIT Campus P. O., Kozhikode 673 601, India \\ ${ }^{2}$ Department of Civil Engineering, Sardar Vallabhbhai National Institute of Technology, Surat 395007 , India
}

\begin{abstract}
This study makes an effort to model service-headway distribution at manually operated toll plazas (MTC) under mixed traffic conditions. To identify the most suitable probability distribution among the selected candidate distributions, Kolmogorov-Smirnov, Anderson-Darling, and chi-squared tests were performed. It was found that the generalized extreme value (GEV) was the most suited distribution for modelling serviceheadway distribution at tollbooths. The results show that GEV distribution parameters can capture possible variations in service headway at tollbooths under MTC reasonably well. The study results can also be used for capacity and level-of-service estimation and the development of warrants for converting MTC to electronic lanes.
\end{abstract}

Keywords: Generalized extreme value, mixed traffic conditions, probability distribution, service headway, shape factor, tollbooth.

IN India, recently, the most accepted mode for highway project execution is under public-private partnership (PPP) scheme. Due to the huge requirement of highway infrastructure and limited Government funding, the PPP component emerges as the most attractive scheme for highway network development. In a PPP project, the government invites private firms to design, build and maintain a road network, with the condition of a predefined time period, after which the facility is transferred to the Government for maintenance. During a predefined period, private firms are authorized to collect toll charges from the road users to recover their capital investment. As a part of PPP projects, toll plazas are built along with highway networks to facilitate collection of toll from the users. There exist broadly three methods for toll collection-manual toll collection (MTC), automated coin machine (ACM), and electronic toll collection (ETC). In an emerging country like India, generally, the MTC method is used predominantly, partially due to mixed

*For correspondence. (e-mail: sarkatkar@gmail.com) traffic conditions and the lack of reliable automation in tracking vehicles with adequate accuracy, in particular. Mixed traffic conditions prevailing on all types of roads in India, are also observed at toll plaza locations in different parts of the country (Figure 1). A dedicated lane is provided at the toll plazas for each vehicle category, but drivers usually select a lane which they perceive to have the least delay. This leads to non-lane-based mixed traffic conditions with poor lane-discipline. This, in turn, affects the time-headway adversely and hence the capacity of the tollbooth, thereby causing an increase in waiting time in the queue at the toll plaza for road users. Conventionally, headway is defined as the time difference between two consecutive vehicles at a designated test point, measured from the same reference point for both vehicles. In this study, at toll plazas, service headway is defined as the difference in exit times of two consecutive vehicles at the same tollbooth measured from a similar reference point for both vehicles. This implies that service headway at the toll plaza includes interarrival time and service time for a given vehicle. Hence, it is well anticipated that under mixed traffic conditions prevailing in developing countries like India, variation in inter-arrival time depends upon vehicle category, pair of leading-following vehicle classes, driver behaviour, and acceleration and deceleration characteristics (which depends on vehicle category and drivers' behaviour). The variation in service time also depends upon various factors such as toll rate, personal attributes of drivers (age, education and experience), personal attributes tollbooth operators (age, gender, working hours, working efficiency and experience), vehicle class and availability of currency change for the toll. It may be interesting to check the most suitable (best-fitted) probability distribution function, which can explain the variation due to numerous aspects, as explained later in this article.

The headway distribution models that are probability density functions (PDFs) have been studied in the past, mainly with objectives associated with an operational analysis of various facilities (for example, highways, urban roads, work-zone roundabouts, signallized and 


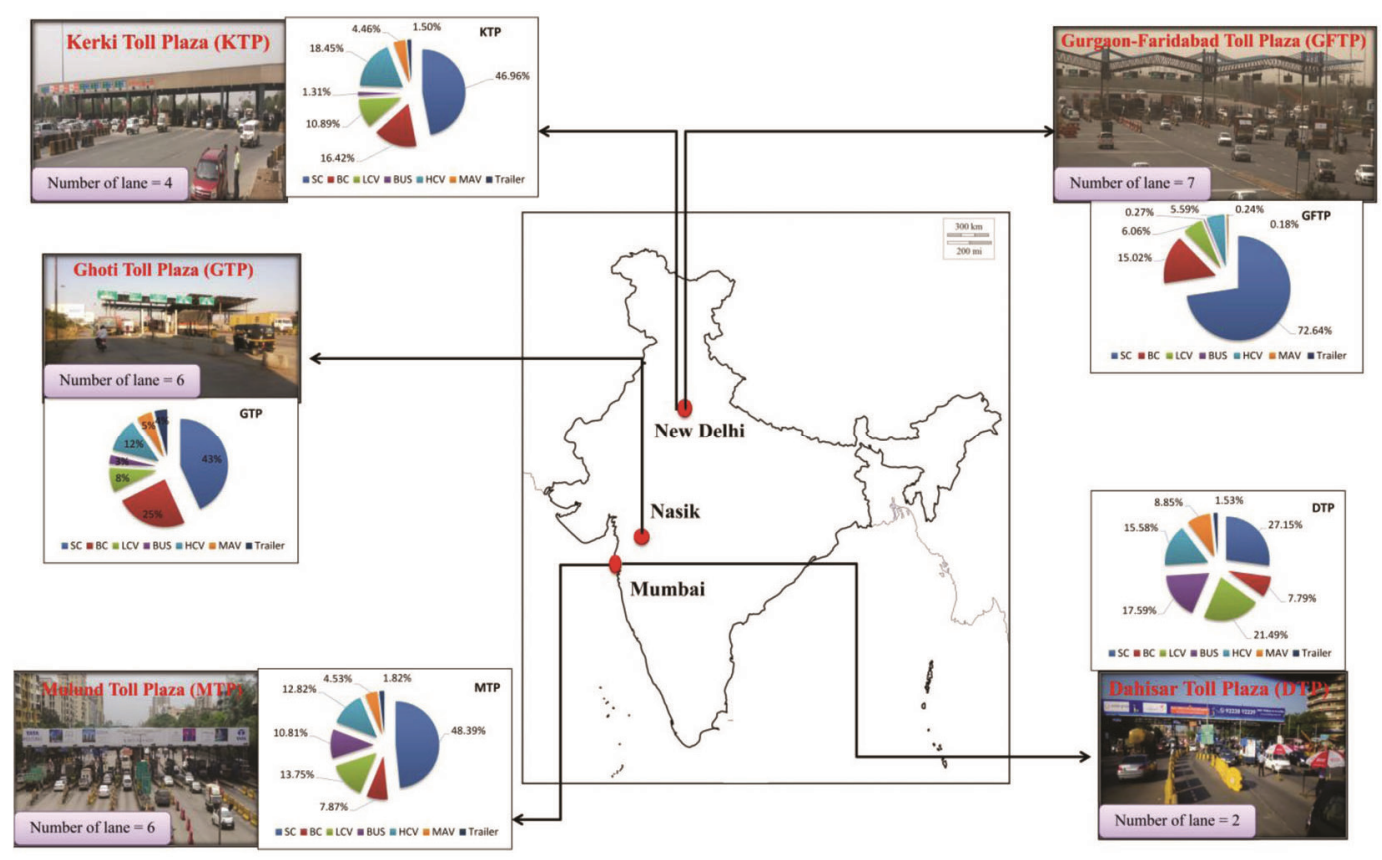

Figure 1. Details of traffic composition observed at selected study sites.

unsignallized intersections), level of service, driver behaviour, vehicle generation in micro-simulation tools and driving simulation applications, among others. Several researchers have studied headway distribution modelling in different scenarios such as highways, work zone, roundabouts, signallized and unsignallized intersections, as well as rural and urban roads ${ }^{1-10}$, but limited studies are available explaining the headway distribution and related traffic characteristics at toll plazas, particularly under mixed-traffic conditions. Currently, a substantial number of toll plazas are operated manually in emerging countries like India. The option of implementing ETC is being considered for better traffic management and efficiency. Presently, however, not much literature is available which may indicate thresholds for conversion of MTC lanes to ETC. Thus, presently, an effort is made to study the manual toll operations as comprehensively as possible, to develop thresholds for implementing ETC under mixed traffic conditions or where similar traffic conditions exist. Hence, the present study is focused on examining different potential service headway distribution models, which may be used in vehicle generation and movement modules of simulation tools. Variation in the most apt suitable probability distribution parameters using traffic data collected at various toll plazas situated in different parts of the country can be useful. This could actually shed light on the causes of variation with reasonable scientific rigour.

\section{Need of the study}

The headway at any facility is the essential traffic flow parameter for estimating the capacity of that facility, simulation studies, safety and driver behaviour studies. The tollbooth capacity is also a function of service headway ${ }^{11-14}$. Thus, to estimate tollbooth capacity, service headway is necessary. Also, in India, traffic consists of more than seven classes of vehicles. These cause heterogeneity in the operational traffic characteristics. On the other hand, driver behaviour at the toll plazas, of joining the shortest queue, causes mixed traffic conditions (i.e. presence of a different class of vehicles) in the dedicated lanes. This hampers the capacity as well ${ }^{15}$. Simulation models without proper knowledge regarding serviceheadway distribution may cause errors in the calibration and, thus, the results. To the best of our knowledge, the headway distributions have been studied for different facilities such as freeways, work zones, urban roads, etc. but not for toll plazas and under mixed traffic conditions. Hence, the present study focuses on service-headway distribution of vehicles at the tollbooths under mixed traffic conditions.

\section{Literature review}

Many studies have reported headway distribution models, as they play a vital role in any simulation model performance ${ }^{16}$. Roadway capacity can be estimated using time headway. Hence, the accurate headway distribution would help maximize road capacity and minimize delay ${ }^{17}$. Furthermore, headway is also useful in research areas related to traffic safety, driver behaviour, and traffic flow theory ${ }^{18}$. Eventually, the headway (probability) distribution study is among the most basic, significant and crucial. Many studies examine the headway distribution in different scenarios, such as freeway, two-lane 
rural roads, signallized and unsignallized intersections, roundabouts, work zone and urban roads. Al-Ghamadi ${ }^{1}$ examined headway distribution characteristics on urban roads in Riyadh Saudi Arabia. He concluded that negative exponential distribution was found to be the best-fit at flow less than $400 \mathrm{veh} / \mathrm{h}$ (low flow), shifted to exponential and gamma distribution for 400-1200 veh/h (medium flow), and Erlang distribution for more than $1200 \mathrm{veh} / \mathrm{h}$ (high flow). Dey and Chandra ${ }^{4}$ examined headway distribution in a steady car-following state on two-lane roads under heterogeneous traffic conditions. They proposed gamma and lognormal distributions for the desired time gap and time headway respectively, under mixed traffic conditions. Riccardo and Massimiliano ${ }^{19}$ reported that inverse Weibull appears to be the most suitable distribution model for most of the flow ranges on rural two-lane roads in Italy. Dubey et al. ${ }^{20}$ proposed generalized pareto (for flow value less than $1500 \mathrm{veh} / \mathrm{h}$ ) and generalized extreme value (GEV) (for flow value more than $1500 \mathrm{veh} / \mathrm{h}$ ). It has also been reported that GEV tends to show better tail modelling properties compared to other conventional distributions used to model vehicular time gap, particularly under mixed traffic conditions. Zhang and $\mathrm{Wang}^{21}$ examined the headway distribution in freeway work zones. They used a non-parametric model with the Gaussian kernel model, and the analysis was done irrespective of the vehicle class. Dong et al. ${ }^{22}$ studied vehicle classspecific headway distribution modelling in a freeway work zone. The results showed that the parametric model with lognormal distribution performed better than a nonparametric model with a Gaussian kernel. Panichpapiboon $^{8}$ studied the headway distribution of vehicles travelling on an urban expressway in Bangkok. He concluded that the GEV distribution model is more effective compared to exponential and lognormal distribution. Yun and Huang ${ }^{9}$ studied the headway distribution at the weaving section on the upstream of signallized intersections. The study concluded that negative exponential distribution performs better in the normal period. Kumar et al. ${ }^{23}$ examined traffic-flow parameters in merging sections and found that the headway follows GEV distribution. Mondal and Gupta ${ }^{24}$ studied headway during queue dissipation at signallized intersections. They found lognormal as the best fit for headway distribution.

As described above, most of the researchers have studied headway distribution for different roadway facilities such as urban roads, rural roads, two-lane roads, work zones, freeways, signallized intersections and roundabouts. Overall, very few headway distribution studies are available on tollbooths, particularly under heterogeneous traffic conditions in the literature. Hence, it is important to study headway characteristics for planning and to improve the efficiency of tollbooths. With this aim the present study has been designed to analyse vehiclespecific and pairwise (only small car (SC) as a leader or follower) headway distribution modelling, applying a statistical approach at different toll plaza locations under mixed traffic conditions.

\section{Basic terminologies used in the present study}

Service headway is defined as the difference in exist times of two consecutive vehicles at the same tollbooth measured from a similar reference point for both vehicles.

A tollbooth is a specific booth where the vehicle stops for transaction. It is a part of the toll plaza facility.

The toll plaza comprises of several tollbooths.

\section{Research methodology}

This study is conducted to determine the best-fitted distribution model for service headway at tollbooths considering the possible variation in flow levels, traffic composition, driver behaviour, geographical location, and of manually operations using traffic data from five toll plaza locations under mixed traffic conditions in India. The data collected at five different locations were used for analysis to capture diversity in the behaviour of driver and tollbooth operators under different traffic environments such as urban and rural roadway facilities. The flow variation was observed to be between 64 and $212 \mathrm{veh} / \mathrm{h}$ at a tollbooth lane, signifying that all possible variations are well-captured through this experimental design. In the present study, a statistical tool, viz. EasyFit 5.5 has been used ${ }^{25,26}$ to evaluate the goodness-of-fit employing popular tests such as the Kolmogorov-Smirnoff (KS), Anderson-Darling (AD), and chi-squared to obtain the best-fit robust distribution among the potential ${ }^{25}$ distributions selected ${ }^{27,28}$. The null hypothesis for this test is that the data follow a specified distribution, whereas the 'alternate hypothesis' is described as the data does not follow the specified distribution. As hypothesized after a thorough review of past studies related to various roadway facilities, many theoretical distributions were considered initially. Efforts were made to find the probability of no-difference between the two datasets (field-observed data and theoretically modelled data), to determine whether the two datasets were statistically significant. As service headway is a continuous entity, several potential continuous distributions were tested initially. After careful observation, the following distributions - lognormal, inverse Gaussian, GEV and Wakeby (Table 1) were found to fit the data set reasonably well. However, GEV distribution was found to offer better results compared to the others distribution. Hence, in the present study, GEV distribution was selected to model the service-headway distribution at manually operated toll plazas under the prevailing mixed traffic conditions. This is how it is treated as a random variable ${ }^{29}$, explaining which could give a certain idea about the sensitivity of a variable considered for study. In uncertainty data analysis (modelling 
RESEARCH ARTICLES

Table 1. Probability density functions (PDF) and CDF for selected distributions

\begin{tabular}{ll}
\hline Distribution & PDF \\
Lognormal & $f(x)=\frac{\exp \left(-\frac{1}{2}\left(\frac{\ln x-\mu}{\sigma}\right)^{2}\right)}{x \sigma \sqrt{2 \pi}}, \quad F(x)=\Phi\left(\frac{\ln x-\mu}{\sigma}\right)$, \\
where $\Phi$ is the Laplace integral.
\end{tabular}

where $\sigma$ is the shape parameter $(\sigma>0)$ and $\mu$ is the location parameter.

Inverse Gaussian

$$
f(x)=\sqrt{\frac{\lambda}{2 \pi(x-\lambda)}} \exp \left(\frac{\lambda(x-\lambda-\mu)^{2}}{2 \mu^{2}(x-\mu)}\right),
$$

where $\lambda$ is the shape parameter $(\lambda>0), \mu$ the location parameter $(\mu>0)$ and $\gamma$ is the continuous location parameter ( $\gamma \equiv 0$ yields the two-parameter inverse Gaussian distribution).

$$
f(x)= \begin{cases}\frac{1}{\sigma} \exp \left(-(1+k z)^{-1 / k}(1+k z)^{-1-1 / k}\right. & k \neq 0 \\ \frac{1}{\sigma} \exp (-z-\exp (-z)) & k=0\end{cases}
$$

where $k$ is the continuous shape parameter, $\sigma$ the continuous scale parameter $(\sigma>0)$ and $\mu$ is the continuous location parameter.

Wakeby

$$
X=\xi+\left(\frac{\alpha}{\beta}\right)\left(1-(1-U)^{\beta}\right)-\left(\frac{\gamma}{\delta}\right)\left(1-(1-U)^{-\delta}\right),
$$

where $U$ is the standard uniform random variable, $\beta, \gamma$ and $\delta$ are the shape parameters, and $\alpha$ and $\xi$ are the location parameters. where $\Phi$ is the Laplace integral.

$$
F(x)=\Phi\left(\sqrt{\frac{\lambda}{x-\gamma}}\left(\frac{x-\gamma}{\mu}-1\right)\right)
$$$$
+\Phi\left(-\sqrt{\frac{\lambda}{x-\lambda}}\left(\frac{x-\gamma}{\mu}-1\right)\right) \exp (2 \lambda / \mu)
$$

where $\Phi$ is the Laplace integral.

$$
\begin{aligned}
& \qquad f(x)=\left\{\begin{array}{l}
\exp \left(-(1+k z)^{-1 / k}\right), \\
\exp (-\exp (-z))
\end{array},\right. \\
& \text { where } z \equiv \frac{x-\mu}{\sigma}
\end{aligned}
$$

$$
f(x)=\frac{(1-F(x))^{\delta+1}}{\alpha t+\gamma},
$$

where $F$ is the cumulative distribution function $t=(1-F(x))^{\beta+\delta}$. precipitation extremes), GEV shows a better fit than other distributions ${ }^{30}$. Chepuri et al. ${ }^{31}$ showed the variation in travel-time data at different flow levels (uncertainty in travel-time data), wherein travel time, a continuous random variable, was modelled using GEV distribution. Real-field observations suggest a wide variation in service-headway values at the tollbooths, and it is expected that variation in service headway can be well-captured using the shape of the GEV distribution and its associated parameters. To demonstrate this, service-headway data at the five different toll plaza locations were extracted considering various leader-follower pairs (SC as a leader or follower only), traffic composition and traffic flow during different time of the day. The GEV distribution parameter such as shape factor $(k)$ was analysed for possible variation associated with traffic flow characteristics at manually operated toll plazas, thereby unfolding the change phenomenon associated with service headway, is the primary outcome of this study.

\section{Data collection}

Videographic data were collected for the present study from five different locations in the northern and western parts of India, of which two are located in the national capital and one in the economic capital, and another on a
National Highway (Figure 1). The variation in site selection was decided to capture the wide variety of traffic flow characteristics, and the behaviour of driver and tollbooth operator. Ghoti (L1) toll plaza is located on National Highway-3 (NH-3) near Nasik, Maharashtra, the Gurgaon-Faridabad (L2) and Kerki (L3) toll plazas are located in the national capital region (NCR), and Mulund (L4) and Dahisar (L5) toll plazas are located in Mumbai, Maharashtra. The data were collected mainly during spring season under clear weather conditions in different phases. Data collection was carried out on both weekdays and weekends during morning and evening peak hours, when there is continuous queue at the tollbooths. As service headway is a continuous phenomenon under queuing conditions, an effort was made to collect data only when a continuous queue was present at the tollbooths. Overall, from traffic-flow characteristics, seven vehicle categories were classified based on their physical and operational characteristics (Figure $2 a)^{15,32}$. Vehicle length is given importance due to the nature of serviceheadway measurement in a given toll-lane. The serviceheadway distributions at tollbooths were studied over a wide range of moderate and under congested traffic-flow conditions (64 to $212 \mathrm{veh} / \mathrm{h}$; Figure $2 \mathrm{~b}$ ). It is observed from Figure $2 b$ that during different time-periods, the volume changes according to traffic composition and thus capturing possible variation in traffic at toll plazas. 


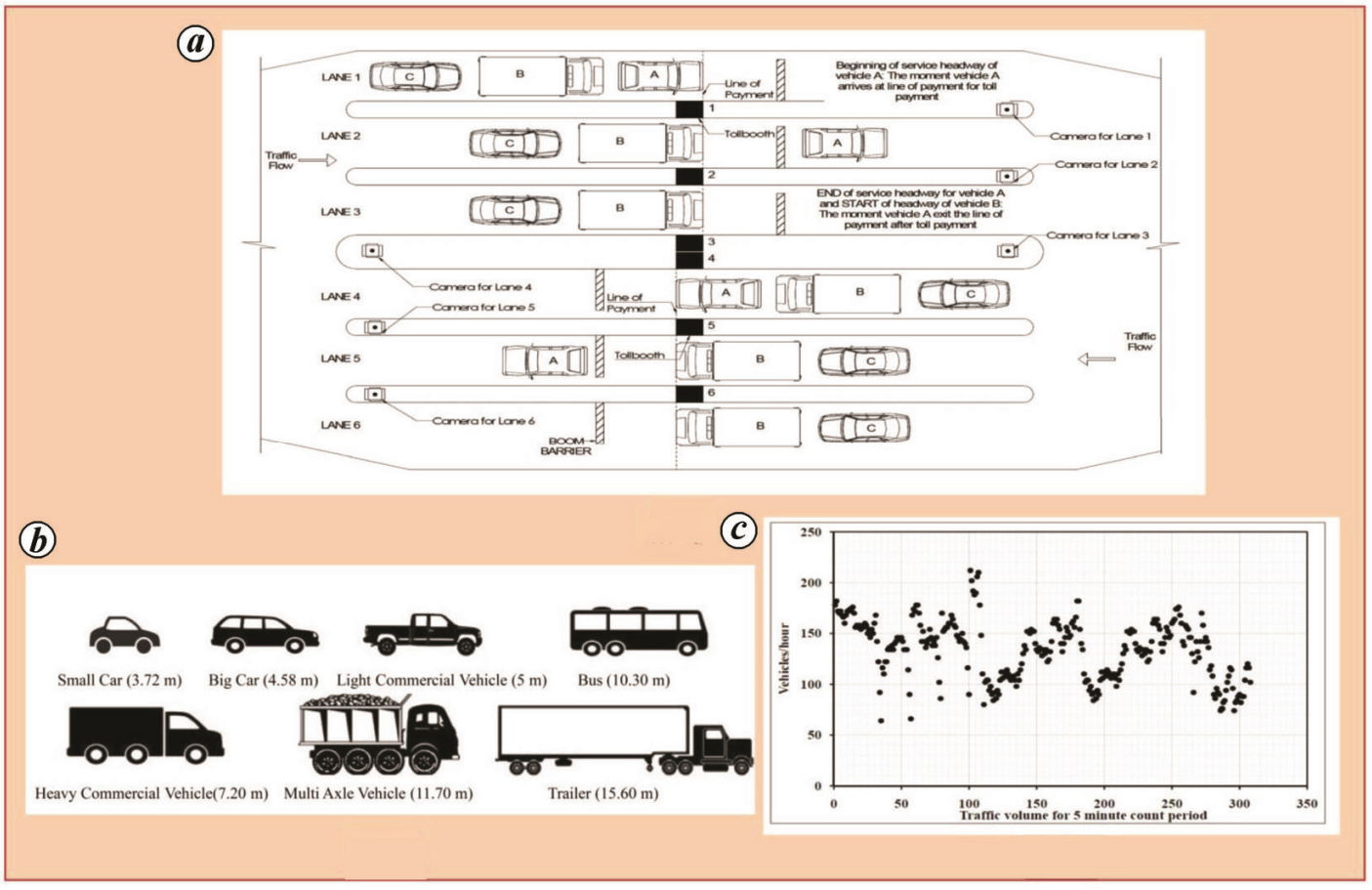

Figure 2. Variation in vehicle counts at selected study location: (a) Graphical representation of service headway terminology, (b) Vehicle class and (c) Variation in traffic volume count for every 5-minute period.

Data connected with all the traffic-flow characteristics were extracted for 25 lanes across five different toll plazas by replaying the video on a large-screen monitor in the laboratory. To achieve the desired degree of precision, the time was noted up to two decimals of seconds extracted using AVIDEMUX 2.6 player. In the worksheet, data such as location, lane, vehicle category, vehicle entry and exit times at the tollbooth (exactly at the toll window for the transaction), type of leading, and following vehicle pairs were entered. Figure $2 a$ is a schematic representation of the service headway at the tollbooths.

\section{Analysis of traffic composition}

It was observed that lane nos 1, 2 and 4 were dedicated mainly for cars-only traffic to pay toll at location L1. However, other vehicle categories were also found in these lanes which were designated for car-traffic only. Alternatively, at location L2, cars were found to be present in the car-only designated lanes, and a combined share of small and big cars was observed to be more than $90 \%$; hence, the traffic condition was found to be homogenous. For locations L3, L4, and L5, mixed traffic conditions were observed in all the lanes. The proportion of heavy vehicles, for example, buses ranged from $0.35 \%$ to $17.30 \%$ whereas, the proportion of heavy commercial vehicle, mainly comprising heavy trucks were found to range between $6.18 \%$ and $15.34 \%$ across different study locations. Figure 1 shows the traffic composition of different vehicle classes observed at the toll plazas.

\section{Descriptive statistics for service-headway analysis at tollbooths}

It is observed from real-field data that service headway varies substantially across different vehicle categories with varying locations and diversity in pairwise combinations (leader-follower pairs). To capture these variations in service headway, vehicle class, and location-wise boxplots were drawn using extracted data (Figure $3 a-f$ and Table 2). Table 2 presents the vehicle category-wise descriptive analysis, such as the number of samples, minimum, maximum, mean service headway values, standard deviation, skewness, and coefficient of variation (CV). From Table 2, it is observed that the number of SC observed is the maximum, followed by big car (BC) and other vehicle categories. The average service headway for $\mathrm{SC}$, for example, is found to be about $15.04 \mathrm{sec}$, whereas, for bus and multi axle vehicle, it is found to be $26.29 \mathrm{sec}$ and $35.45 \mathrm{sec}$ respectively. The minimum and maximum service headway for trailers is $14.36 \mathrm{sec}$ and $40.31 \mathrm{sec}$ respectively $\mathrm{SC}$, as a leader or follower vehicle combination (vehicle pair combinations), shows wide variation in service headway values. For SC following SC, the mean service headway is $14.63 \mathrm{sec}$, whereas for SC following a trailer, it is $39.88 \mathrm{sec}$. The field observations strongly suggest that service headway of any vehicle at a tollbooth is not constant, but varies over a wide range (Figure 3 ).

The observed value of service headway shows that under mixed traffic conditions at tollbooths, the service headway is more than $15 \mathrm{sec}$, higher than that specified 

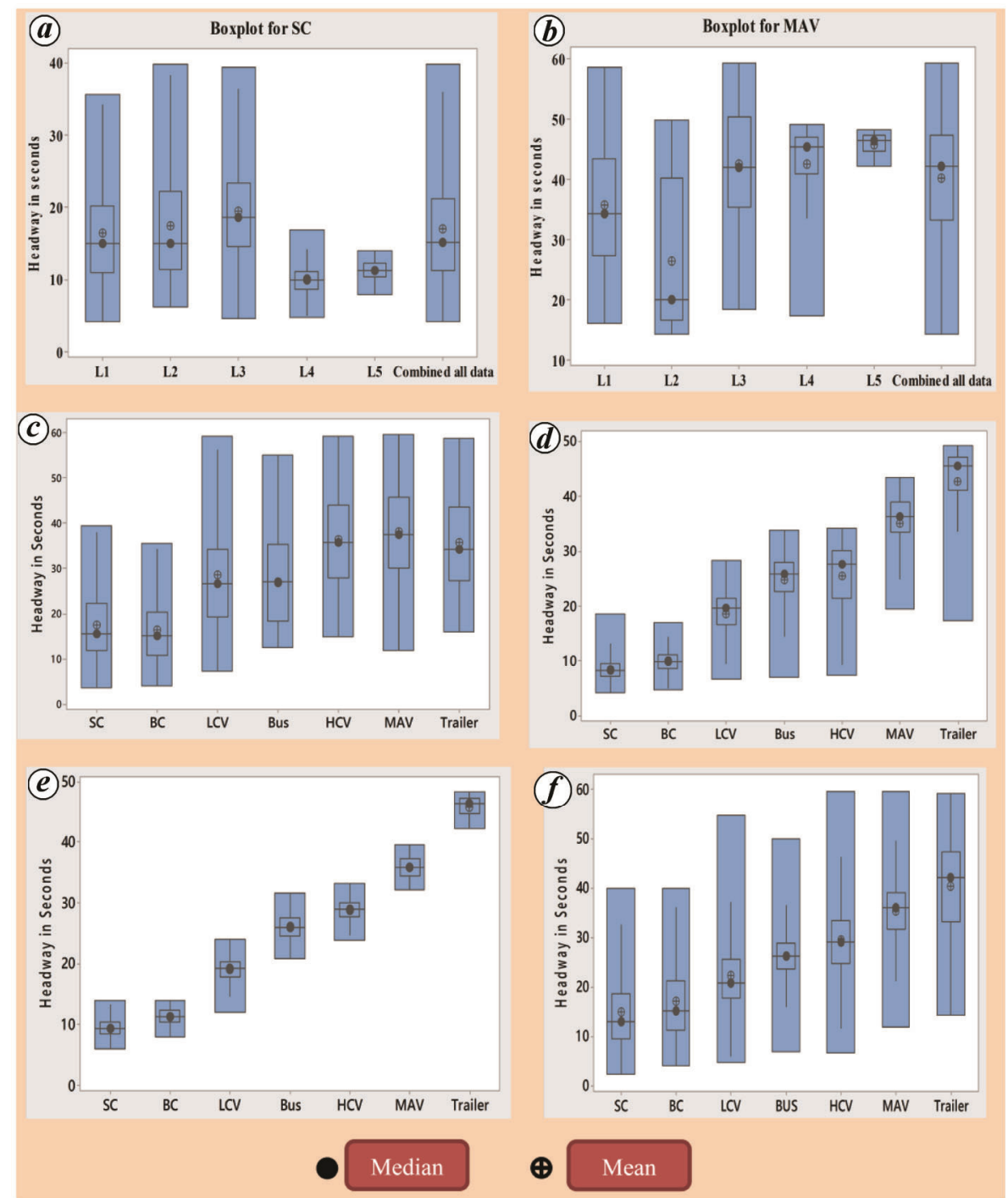

Figure 3. Vehicle category wise variation in service headway from real field data: $\boldsymbol{a}, \mathrm{SC} ; \boldsymbol{b}, \mathrm{MAV} ; \boldsymbol{c}, \mathrm{L} 1 ; \boldsymbol{d}, \mathrm{L} 4 ; \boldsymbol{e}, \mathrm{L} 5 ; \boldsymbol{f}$, combined all data.

by the Indian Road Congress (IRC) ${ }^{33}$. In general, according to the guidelines mentioned, it should not be more than $15 \mathrm{sec}$ irrespective of the vehicle category. This variation in service headway may be attributed to the mixed traffic conditions, vehicle static and dynamic characteristics, the varying vehicle class-wise toll rates of personal attributes of drivers and tollbooth operators and random arrival of vehicles at the tollbooths.

From Table 2, it may be noted that the mean service headway value for $\mathrm{SC}$ and $\mathrm{BC}$ is closer to a mean value in comparison to heavy vehicles. It may also be noted that $\mathrm{CV}$ is higher for SC and BC (more than 0.4) in comparison to that estimated for heavy vehicles. The minimum and maximum values obtained are observed to vary as a function of vehicle category and its characteristics. Similar observations can also be realized after a careful examination of descriptive statistics for different vehicle pairs, wherein SC is either a leading or a follower vehicle. The mean service headway values are found to be substantially lower for vehicle pairs when SC acts as a leading vehicle, in comparison to vehicle pairs when SC acts as a following vehicle. However, for $\mathrm{CV}$, the results are exactly reversed, which may be attributed to the manoeuvrability of vehicles and drivers behaviour when $\mathrm{SC}$ is present as a leading or following vehicle.

\section{GEV distribution analysis}

The empirical service headway distribution associated with each vehicle class and also for different leaderfollower (only when SC is a leader or follower) vehiclepair combinations observed fit well using GEV distribution. This was confirmed using robust tests such as KS, $\mathrm{AD}$, and chi-squared to evaluate the goodness-of-fit. Table 3 provides a vehicle- specific summary of GEV 
RESEARCH ARTICLES

Table 2. Descriptive analysis of service headway

\begin{tabular}{|c|c|c|c|c|c|c|c|}
\hline Vehicle class & Sample size & $\begin{array}{l}\text { Minimum } \\
\quad(\mathrm{sec})\end{array}$ & $\begin{array}{l}\text { Maximum } \\
\quad(\mathrm{sec})\end{array}$ & $\begin{array}{c}\text { Mean } \\
(\mathrm{sec})\end{array}$ & $\begin{array}{c}\text { Standard } \\
\text { deviation }(\mathrm{sec})\end{array}$ & $\begin{array}{l}\text { Skewness } \\
\quad(\mathrm{sec})\end{array}$ & $\begin{array}{l}\text { Coefficient of } \\
\text { variation }\end{array}$ \\
\hline Small car (SC) & 6540 & 2.44 & 39.88 & 15.04 & 7.35 & 1.18 & 0.48 \\
\hline Big car (BC) & 1842 & 4.16 & 39.92 & 17.11 & 7.41 & 0.95 & 0.43 \\
\hline $\begin{array}{l}\text { Light commercial vehicle } \\
\text { (LCV) }\end{array}$ & 1350 & 4.68 & 54.84 & 22.45 & 7.90 & 1.21 & 0.35 \\
\hline Bus & 557 & 6.92 & 49.92 & 26.29 & 6.14 & 0.34 & 0.23 \\
\hline $\begin{array}{l}\text { Heavy commercial vehicle } \\
\qquad(\mathrm{HCV})\end{array}$ & 1650 & 6.76 & 59.68 & 29.48 & 8.90 & 0.47 & 0.30 \\
\hline Multi axle vehicle (MAV) & 508 & 11.96 & 59.8 & 35.45 & 7.76 & 0.10 & 0.21 \\
\hline Trailer & 226 & 14.36 & 59.4 & 40.31 & 10.13 & -0.35 & 0.25 \\
\hline All combined & 12673 & 2.44 & 59.8 & 20.42 & 11.01 & 0.99 & 0.53 \\
\hline $\mathrm{SC}-\mathrm{SC}$ & 4128 & 2.44 & 39.76 & 14.63 & 7.32 & 1.20 & 0.50 \\
\hline $\mathrm{SC}-\mathrm{BC}$ & 1005 & 4.16 & 39.92 & 16.64 & 7.37 & 1.00 & 0.44 \\
\hline $\mathrm{SC}-\mathrm{LCV}$ & 483 & 4.68 & 59.28 & 22.27 & 10.09 & 1.20 & 0.45 \\
\hline SC-Bus & 174 & 6.80 & 55.12 & 24.44 & 8.59 & 0.24 & 0.35 \\
\hline $\mathrm{SC}-\mathrm{HCV}$ & 538 & 6.32 & 59.68 & 29.14 & 10.60 & 0.27 & 0.36 \\
\hline SC-MAV & 150 & 8.00 & 59.80 & 33.24 & 9.76 & -0.11 & 0.29 \\
\hline SC-Trailer & 66 & 9.92 & 59.40 & 39.88 & 11.80 & -0.31 & 0.29 \\
\hline $\mathrm{BC}-\mathrm{SC}$ & 1009 & 3.64 & 39.80 & 16.13 & 7.73 & 1.13 & 0.47 \\
\hline LCV-SC & 503 & 6.12 & 38.24 & 15.27 & 6.74 & 1.28 & 0.44 \\
\hline Bus-SC & 150 & 5.24 & 36.92 & 13.34 & 6.45 & 1.45 & 0.48 \\
\hline $\mathrm{HCV}-\mathrm{SC}$ & 548 & 4.24 & 39.88 & 16.43 & 6.76 & 0.96 & 0.41 \\
\hline MAV-SC & 125 & 6.24 & 39.6 & 18.23 & 8.65 & 0.91 & 0.47 \\
\hline Trailer-SC & 72 & 6.28 & 37.92 & 17.95 & 7.09 & 0.81 & 0.39 \\
\hline
\end{tabular}

Table 3. Generalized extreme value (GEV) distribution parameter and results

\begin{tabular}{|c|c|c|c|c|c|c|}
\hline \multirow[b]{2}{*}{ Vehicle class } & \multicolumn{3}{|c|}{ GEV distribution parameter } & \multicolumn{3}{|c|}{ Test results } \\
\hline & $\kappa$ & $\sigma$ & $\mu$ & $\mathrm{K}-\mathrm{S}$ test & A-D test & Chi-squared \\
\hline $\mathrm{SC}$ & 0.135 & 4.9275 & 11.445 & Accepted & Accepted & Accepted \\
\hline $\mathrm{BC}$ & 0.072 & 5.4494 & 13.554 & Accepted & Accepted & Accepted \\
\hline $\mathrm{LCV}$ & 0.052 & 5.6896 & 18.858 & Accepted & Accepted & Accepted \\
\hline Bus & -0.255 & 5.5506 & 24.234 & Accepted & Accepted & NA \\
\hline $\mathrm{HCV}$ & -0.171 & 8.0316 & 26.029 & Accepted & Accepted & Accepted \\
\hline MAV & -0.294 & 7.4316 & 32.894 & Accepted & Accepted & NA \\
\hline Trailer & -0.451 & 10.913 & 37.544 & Accepted & Accepted & Accepted \\
\hline All combined & 0.070 & 8.1045 & 15.137 & Accepted & Accepted & Accepted \\
\hline $\mathrm{SC}-\mathrm{SC}$ & 0.150 & 4.813 & 11.025 & Accepted & Accepted & Accepted \\
\hline $\mathrm{SC}-\mathrm{BC}$ & 0.080 & 5.357 & 13.086 & Accepted & Accepted & Accepted \\
\hline SC-LCV & 0.039 & 7.490 & 17.655 & Accepted & Accepted & Accepted \\
\hline $\mathrm{SC}-\mathrm{Bus}$ & 0.350 & 8.439 & 21.824 & Accepted & Accepted & NA \\
\hline SC-HCV & -0.214 & 10.096 & 25.121 & Accepted & Accepted & Accepted \\
\hline SC-MAV & -0.366 & 9.841 & 30.279 & Accepted & Accepted & NA \\
\hline SC-Trailer & -0.389 & 12.52 & 36.276 & Accepted & Accepted & Accepted \\
\hline $\mathrm{BC}-\mathrm{SC}$ & 0.127 & 5.251 & 12.355 & Accepted & Accepted & Accepted \\
\hline LCV-SC & 0.127 & 4.531 & 12.012 & Accepted & Accepted & Accepted \\
\hline Bus-SC & 0.200 & 3.891 & 10.143 & Accepted & Accepted & Accepted \\
\hline $\mathrm{HCV}-\mathrm{SC}$ & 0.042 & 5.125 & 13.253 & Accepted & Accepted & Accepted \\
\hline MAV-SC & 0.086 & 6.278 & 14.027 & Accepted & Accepted & Accepted \\
\hline Trailer-SC & 0.044 & 5.464 & 14.555 & Accepted & Accepted & Accepted \\
\hline
\end{tabular}

NA, Not applicable.

distribution parameters such as shape $(\kappa)$ scale $(\sigma)$ and location $(\mu)$ supplemented with the results of KS, AD and Chi-squared tests for headway distribution at tollbooths under mixed traffic conditions. The decision to accept or reject the null hypothesis is made by comparing the $P$-value at a $5 \%$ level of significance. The null hypothesis is formulated as extracted service headway data follow the GEV distribution. The result shows that the null hypothesis is accepted (data follow the GEV distribution) in all individual vehicle classes, including each of the selected vehicle pair combinations, by applying $\mathrm{KS}$ and $\mathrm{AD}$ tests. In the case of chi-squared test, the null 
RESEARCH ARTICLES
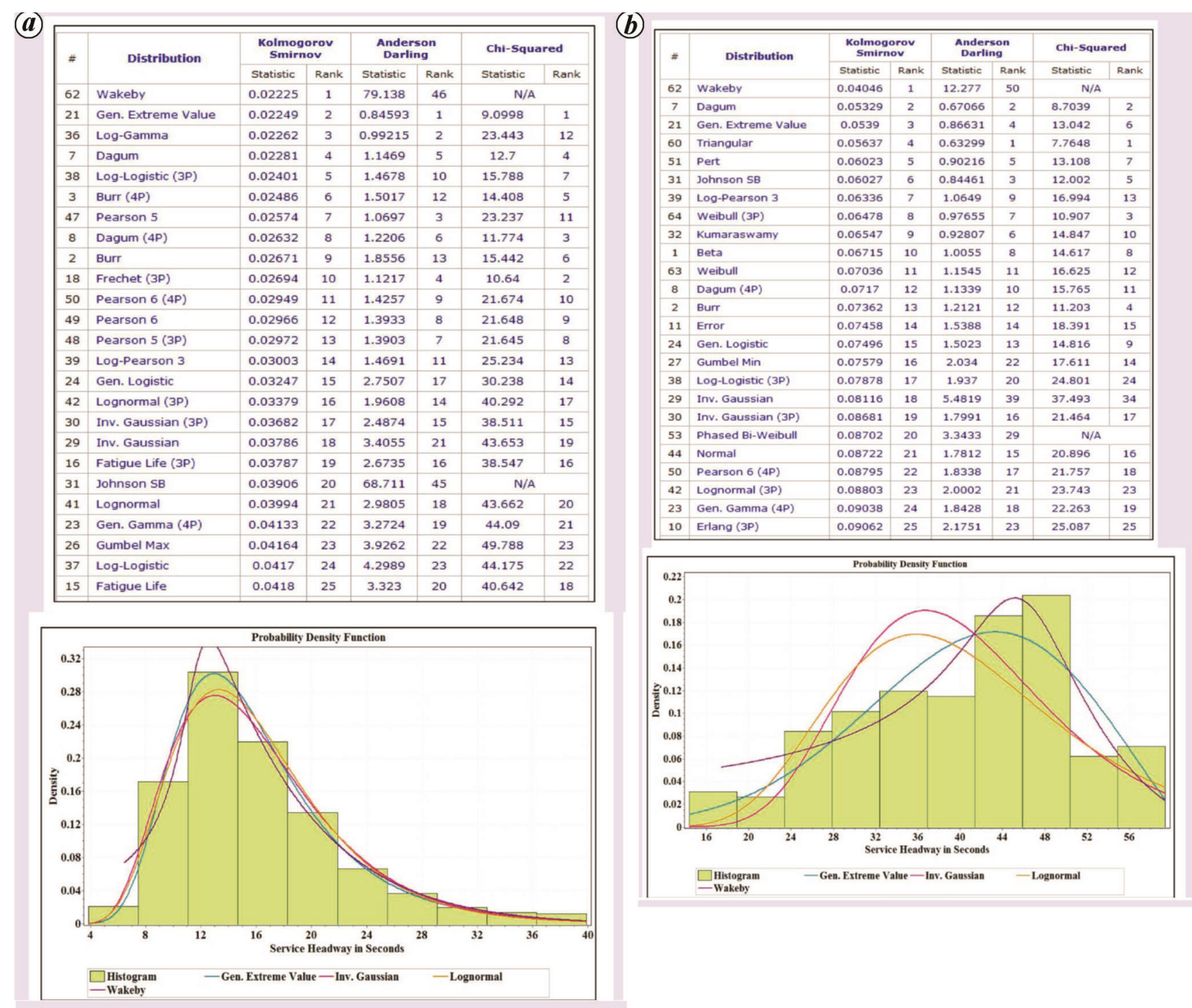

Figure 4. Sample plots for goodness of fit for service headway: $\boldsymbol{a}, \mathrm{SC} ; \boldsymbol{b}$, Trailer.

hypothesis for Bus, MAV, SC-Bus, and SC-MAV is rejected, whereas in all other cases it is accepted. From the results, it may be concluded that overall, GEV shows a better potential to fit all possible variations using goodness-of-fit tests such as $\mathrm{KS}, \mathrm{AD}$, and chi square.

Figure $4 a$ and $b$ shows easy fit output for SC and Trailer respectively, for best fitting distributions along with the PDF curves. From figure, it is concluded that GEV shows the best fitting distribution among all hypothesized distributions in the present study. Further, it would be interesting to check the ability of the GEV distribution to capture variations in service headway due to diversity in locations of toll plazas, traffic composition of vehicles in the queue, and flow levels at different periods. It is speculated that the GEV distribution parameter such as shape factor reasonably explain the possible distribution of random variable, i.e. service headway at manual operated tollbooths under prevailing mixed traffic conditions.

\section{Location-wise analysis of GEV distribution}

Figure $5 a$ through $g$ shows vehicle class-wise variation in the shape factor $\kappa$ for all five toll plaza locations, including for combined data. Location L1 shows a shape factor which is observed to change from near to 0 to -0.25 (Figure $5 a$ ). A shape-factor value near zero implies that service headway for that particular vehicle class is distributed normally. Figure $5 a$ shows that as average service headway and standard deviation increase, shape factor changes from positive to negative, i.e. from higher (positive) to lower (negative) value. The positive sign of shape factor indicates that maximum number of samples fall in the lower service headway range (left-skewed data), whereas the negative sign indicates that the number of observations falls in the higher service headway range (right-skewed data). The results obtained in connection with location L1 confirm that as vehicle length increases, 


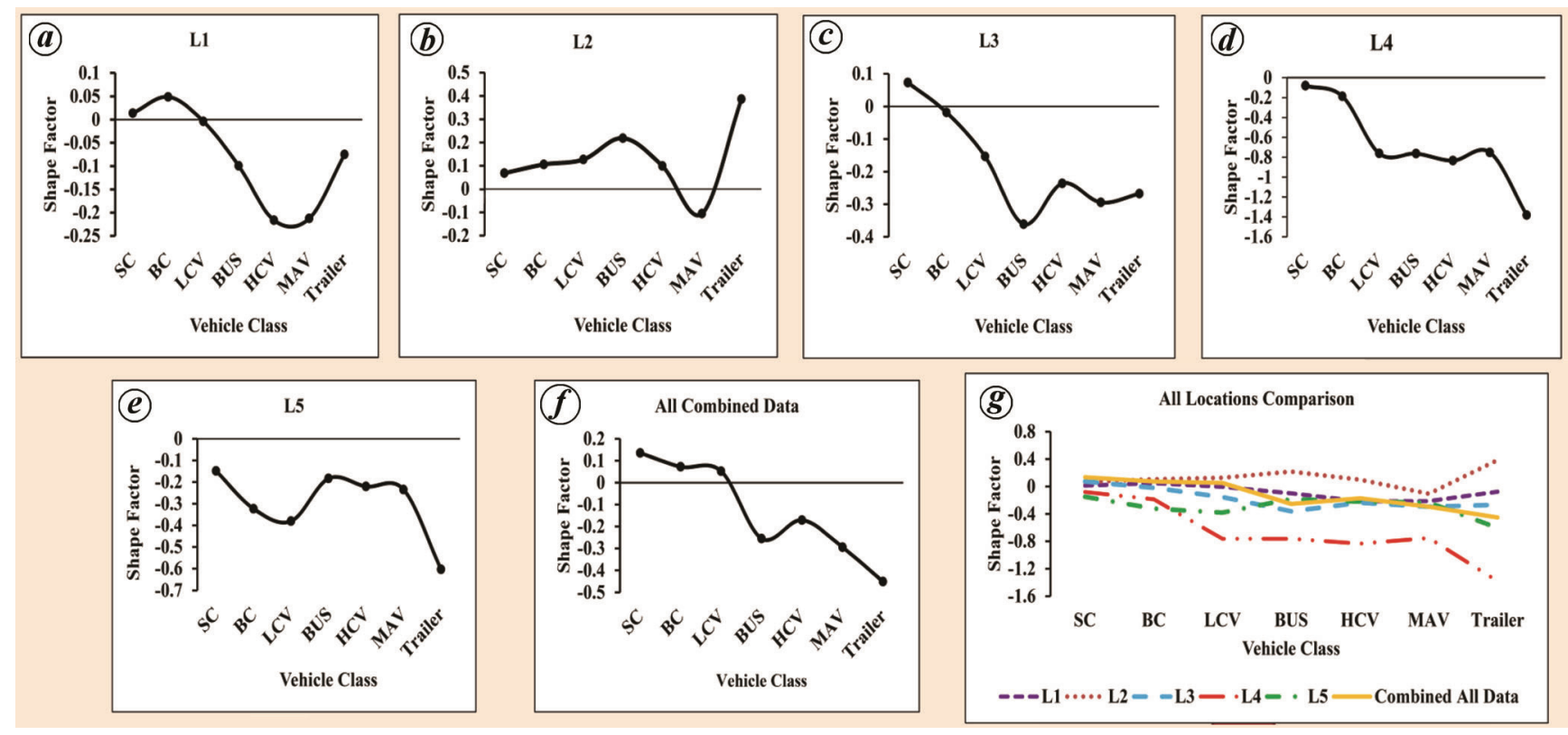

Figure 5. Location wise comparison of GEV shape factor: $\boldsymbol{a}, \mathrm{L} 1 ; \boldsymbol{b}, \mathrm{L} 2 ; \boldsymbol{c}, \mathrm{L} 3 ; \boldsymbol{d}, \mathrm{L} 4 ; \boldsymbol{e}, \mathrm{L} 5 ; \boldsymbol{f}$, combined data; $\boldsymbol{g}$, all locations.

service headway also tends to increase. This can be explained well on the basis of vehicle length. As the vehicle length increases, more clearance time is required. Also, a higher toll amount requires more transaction time (service time). For SC, BC and LCV, more samples are concentrated near lower service headway values, resulting in a positive shape factor, whereas heavy vehicle samples concentrated near a higher service headway range (right-skewed data) show a negative sign. At location L1, SC and LCV show shape factor near zero, indicating that service headway samples for this class are normally distributed.

Figure $5 b$ shows a positive shape factor for all vehicle classes, except MAV at location L2. This toll plaza is located on the daily commuters' route, and SC and BC dominate more than $90 \%$ of the traffic composition share. Hence, all vehicle classes (except trailer) show service headway measurements that fall in the lower range (shape factor is positive), and data are found to be normally distributed (shape factor near zero). Figure $5 c-f$ also suggests changes in the shape factor for locations L3-L5, which was confirmed after careful examination. Location L3 shows a positive shape factor for SC and BC, whereas it gives a negative shape factor for the remaining vehicle classes. Locations L4 and L5 are located at the entry point of Mumbai, and hence the proportion of heavysized vehicles (Bus, HCV, MAV and Trailer) present in the traffic mix is significantly more compared to other locations. Locations L4 and L5 show a negative shape factor for all vehicle categories, which means maximum service headway observations fall in higher ranges for all vehicle classes. This is due to the higher proportion of large-sized vehicles, as observed in the traffic mix sup- plemented with the random arrival of heavy vehicles at tollbooths, which results in maximum service headway observations falling in the higher range. Figure $5 \mathrm{~g}$ shows a comparison of GEV parameters, particularly shape factor for all the locations simultaneously in a single plot. Location L4 depicts maximum number of heavy-sized vehicles (Bus, HCV, MAV and Trailer), which is attributed to maximum service headway observations falling in the higher range compared to other locations, and hence shows maximum negative shape factor values compared to other locations (Figure $5 \mathrm{~g}$ ). The result shows that GEV distribution gives the best-fit across all locations, and a similar result is confirmed for all combined data. The result show that GEV distribution parameters, particularly shape factor, can capture the all possible variations present in the traffic mix by virtue of their effect on service-headway distribution at tollbooths operating under mixed traffic conditions.

\section{Characterizing variation in service headway due to change in vehicle pairs}

The field data observed from videographic survey show that mixed traffic condition prevails at toll plazas. This can be attributed to the random arrival of vehicles of different categories. Moreover, users under mixed traffic conditions select a lane based on their perception (least delay) instead of the allocated dedicated lanes. These create mixed traffic conditions with variation in traffic composition, as the same tollbooth may be used by different vehicle categories, irrespective of its dedicated use. In the present study, due to mixed traffic conditions, 49 

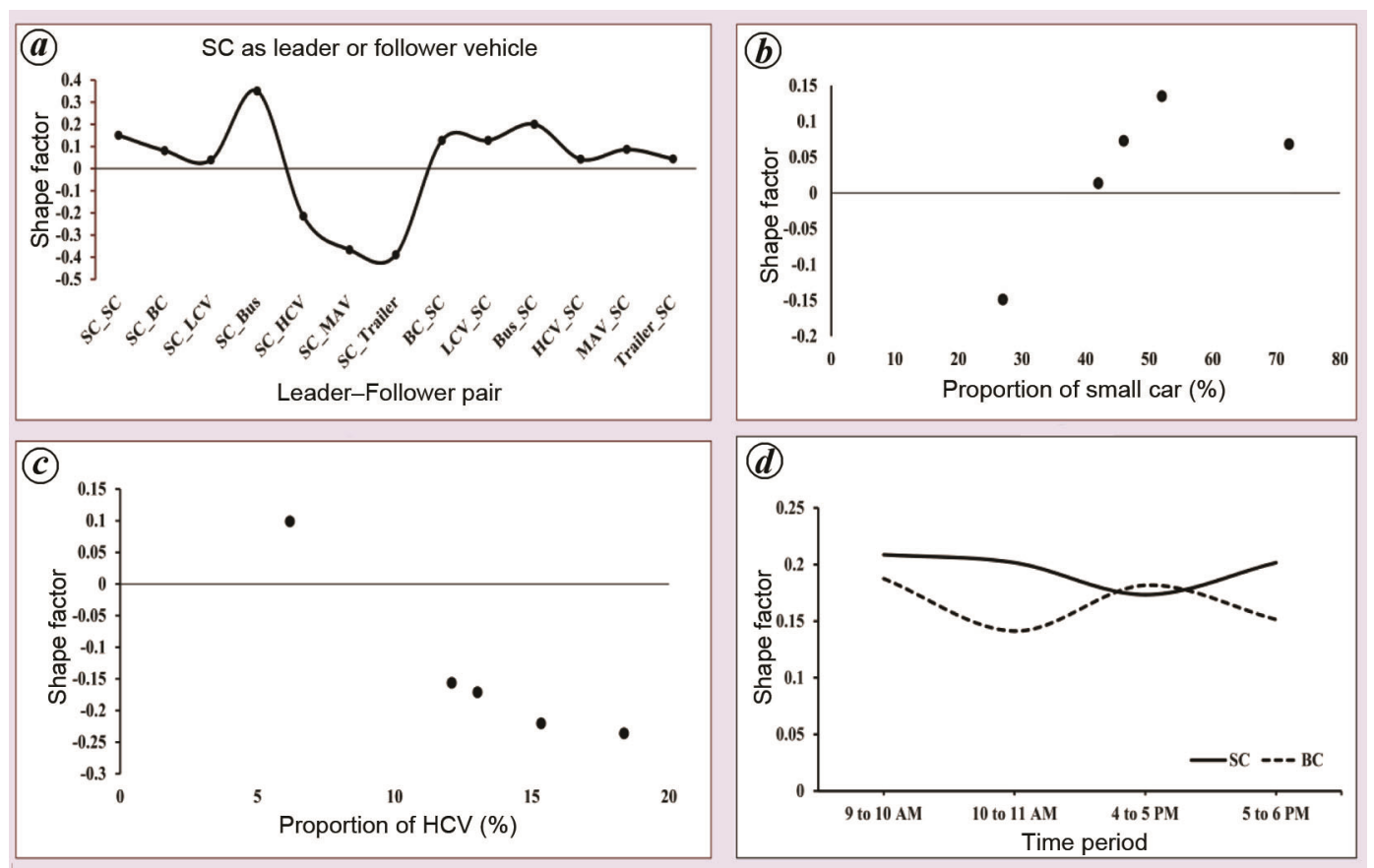

Figure 6. Variation in shape factors: $\boldsymbol{a}, \mathrm{SC}$ as leader/follower; $\boldsymbol{b}$, Effect of SC proportion; $\boldsymbol{c}$, Effect of HCV proportion; $\boldsymbol{d}$, Comparison of GEV shape factor for SC and $\mathrm{BC}$ over a time period.

leader-follower pairs were observed in the field, of which, $\mathrm{SC}$ as a leader or follower pairs, comprising of possible 13 vehicle pairs, are considered as an example. Figure $6 a$ shows comparison of shape factors for 13 pairwise combinations. The result shows that in the case of vehicle pairs, comprising heavy vehicles (HCV, MAV, Trailer) as the follower show the shape factor is negative, which implies that the maximum number of observations fall under higher service headway values. On the other hand, in the case of pairs comprising $\mathrm{SC}$ or $\mathrm{BC}$ as the follower vehicle, the values of shape factor are observed to be positive, which means that the maximum number of observations belong to a lower range of service headway values and near zero; and hence are observed to be normally distributed. This variation may be explained based on the variation of operational manoeuvrability and toll rate value for heavy vehicles. Hence, considering traffic composition data in a given season or a given day of the week, practitioners can forecast variation in service headway using GEV and its shape parameter, which in turn can be used to devise an optimal policy to reduce the operational delay by taking appropriate measures, particularly when heavy vehicles are more in the traffic composition.

\section{Effect of composition on shape factor}

The variation in service headway due to traffic composition was also studied using the shape factor of GEV distribution (Figure $6 b$ and $c$ ). As the proportion of SC increases in the traffic stream from $27 \%$ to $53 \%$, the shape factor tends to move from a negative $(-0.15)$ to a positive $(0.15)$ value. It may be interpreted from Figure $6 b$ that for a lower proportion of SC (less than 30\%) at the tollbooths, the shape factor shows a higher range of service headway values (data are right skewed). Whereas as the SC proportion (more than 30\%) increases the shape factor shows a lower range of service headway values (data are left skewed). This may be attributed to a lower proportion of SC present at a given tollbooth, which indicates greater probability that vehicle-pair combinations contain SC as a follower and any other vehicle category as a leader. In other words, the mixed traffic conditions observed yield a wide range of service headway values, and the maximum observations are concentrated near a higher range of service headway values. Alternatively, if SC proportion increases at a particular tollbooth, there is greater probability that homogenous conditions will prevail. This leads to a traffic situation wherein mostly SC follows SC, and hence vehicle pair combinations tend to generate a maximum number of service headway values in the lower range. After a certain proportion of SC (more than $72 \%$ ) in the observed traffic stream data, the shape factor tends to move towards zero, which indicates that service headway values are normally distributed. This phenomenon can be explained as follows: as share of SC increases in the traffic stream (more than $72 \%$ ), traffic interactions are more homogenous and hence deviate towards normal distribution.

Similarly, Figure $6 c$ shows that as a proportion of $\mathrm{HCV}$ increases in the traffic stream, the shape factor 
changes from a positive $(0.10)$ to a negative $(-0.25)$ value. This indicates more heterogeneous traffic conditions, and hence service headway measurements also deviate away from the normal distribution. This may be explained as follows: as the proportion of $\mathrm{HCV}$ changes from $5 \%$ to $20 \%$, there is greater chance of combinations comprising other vehicle types. This may result in the maximum number of service headway observations on the higher side and hence result in a negative shape factor.

\section{Effect of temporal variation on shape factor}

The influence of temporal variation on shape factor was also examined. The four datasets involving SC and $\mathrm{BC}$ vehicle categories were collected over different periods. Two time periods were selected in the morning and two in the evening. The extracted dataset was then fitted using GEV distribution, and shape factors were estimated. The shape factors for SC and $\mathrm{BC}$ showed positive sign indicating that maximum service headway observation is concentrated near the smaller values (Figure $6 d$ ). The result shows no significant difference between the estimated shape factors for $\mathrm{SC}$ and $\mathrm{BC}$ at different data points. So, it may be concluded that GEV distribution can model service headway distribution at toll plazas under mixed traffic conditions over different periods.

\section{Model validation}

To validate the proposed model (GEV) in the present study, $20 \%$ of datasets were retained before modelling. These datasets were used to examine the transferability of the model. The data extracted for the pairwise combination of LCV-SC contained a total of 165 observations.

Field observations were used for service headway boundaries and to get the expected frequency. The $P$ value was obtained using shape, scale and location parameters $(\kappa, \sigma, \mu)$ for LCV-SC are (from EasyFit) and multiplied with $N$ number of samples. The chi-square test was applied to field-observed and expected frequency samples obtained from the proposed model. For validation purpose, the null hypothesis was formulated that the observed and predicted distributions are the same. The result shows that at the $1 \%$ level of significance at seven degrees of freedom the critical value is 18.47 which is greater than the computed value of 16.20 . Hence, there is no significant difference between the observed and expected frequency values. The chi-square test provides statistically significant evidence, at $99 \%$ confidence level, that the model could be employed at different locations. Further, a two-sample KS test was performed at $1 \%$ level of significance, and results revealed that both the samples belonged to the same distribution type $\left(D_{\text {stat }}\right.$ value as 0.51 with $D_{\text {crit }}$ value of 0.85 ).
We used the quantile-quantile $(Q-Q)$ plot for further validation. The plots are better for GEV distribution and hence were used in the present study ${ }^{34,35}$. The expected versus predicted quantiles values of service headway were plotted. If the points are on or close to $45^{\circ}$ lines (i.e. $X=Y$ ), the distribution is a best-fit. In Figure 7, shows the GEV distribution. The points lie mostly on the $X=Y-$ axis. Hence, the GEV distribution is best fitted for the headway at the tollbooths under mixed traffic conditions.

\section{Summary and conclusion}

Most of the earlier studies modelled time-headway for freeways, urban roads, rural roads, including junctions such as roundabouts and signallized intersections. However, studies on service headway distribution at toll plazas are rather limited and hence, need thorough analysis. The present study examined service-headway distribution characteristics at manually operated tollbooths under prevailing mixed traffic conditions. The results show wide variation in service headway across different vehicle categories and at different locations. This observed variation may be attributed to the manoeuvrability of different vehicle categories (by virtue of size and dynamic characteristics), different vehicle-pair (leaderfollower) combinations, traffic composition, toll rate and personal attributes of driver tollbooth operators.

In this study, $\mathrm{KS}, \mathrm{AD}$ and chi-squared tests were used to examine whether the hypothesized analytical distributions could describe the empirical distribution of data points. Although lognormal, inverse Gaussian and Wakeby distributions were found to reasonably fit well, the GEV distribution outperformed in most of the cases. The GEV parameters (shape, location and scale) also captured the entire service headway variations at the tollbooths most accurately. This corroborates that GEV is the most suitable probability density function for explaining the possible variations in service headway at toll plazas under mixed traffic conditions.

Furthermore, the shape factor of GEV is well explored to describe service headway characteristics at manually operated toll plazas. Interestingly, the variation in shape factor values was also scrutinized for different vehicle categories across different toll plazas, traffic compositions, leader-follower pairs (only for SC as a leader or follower), and periods. It has been well demonstrated that positive shape factor values indicate that service headway values fall in the lower range $(5-20 \mathrm{sec})$. In contrast, negative shape factor values indicate that maximum service headway observations fall in the higher range (30$45 \mathrm{sec}$ ). Shape factor near zero depicts that service headway data are normally distributed, which shows that the mean of service-headway observations falls in the range 20-30 sec (irrespective of vehicle type). In the case of small-sized vehicles (such as SC, BC and LCV), the 


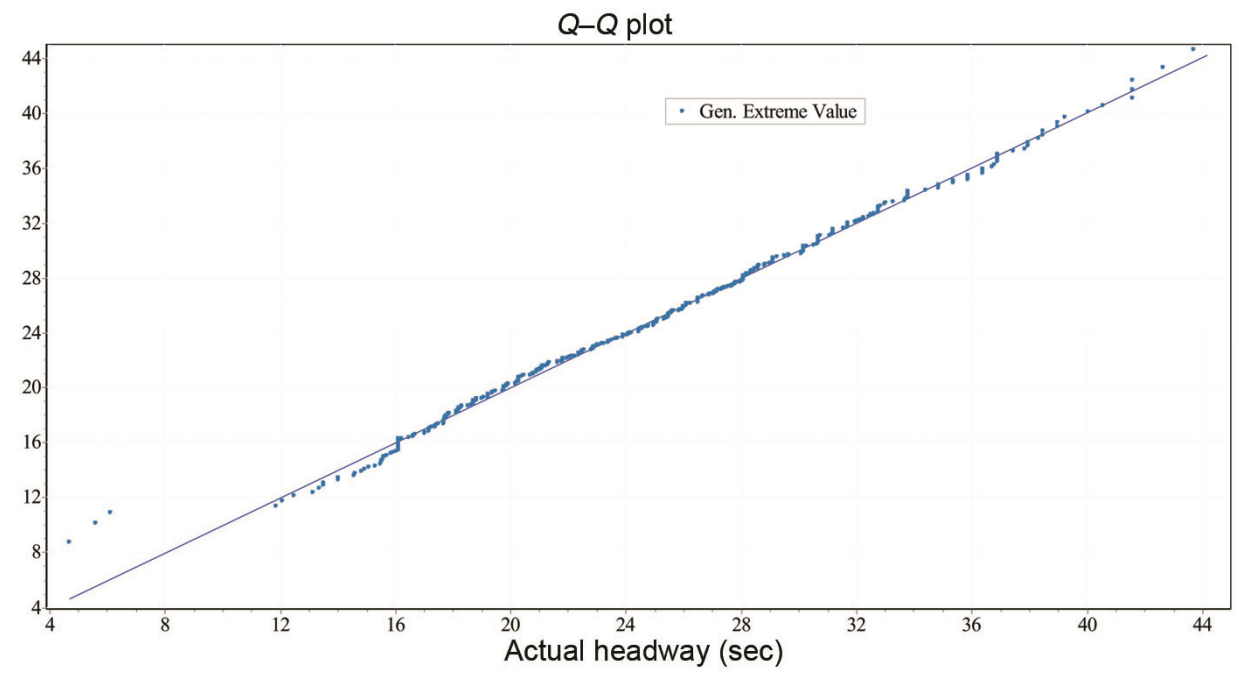

Figure 7. Sample Q-Q plot for service headway considering GEV distribution.

shape factor is found to be positive or near zero, whereas for heavy vehicles (such as Bus, HCV, MAV and Trailer) it shows a negative value for most of the locations.

In summary, this study adds some inferences to the body of literature related to the modelling of vehicle class-wise service headway variations at manually operated toll plazas under mixed traffic conditions, which depicts the situation in most of the developing countries, particularly in the South Pacific Asian countries with automation rarely implemented for monitoring highways due to the prevailing mixed traffic conditions. The shape factor values of GEV distribution may not be directly serviceable at other toll plaza locations. However, the modelling approach presented here certainly holds reasonable promise for extending the proposed concept to other locations in different countries. Certainly, there can be variations in results due to geographical attributes and the behaviour of drivers and tollbooth operators at toll plazas. The outcome of this work also offers a perspective on GEV distribution for explaining the possible variation in service headway that can be used effectively in modelling service-headway distribution at toll plazas. The results of this study may help in good value for developing applications, particularly simulation models for better traffic management in toll plazas. The present study may be useful for tollbooth capacity evaluation, level-of-service based on service headway as a variable, and the development of thresholds for the conversion of MTC to ETC lane. Additionally, introducing the concept of congestion pricing/dynamic tolling strategy at the toll plazas based on service headway variation can be a good policy intervention under mixed traffic conditions prevailing in the developing countries, particularly in South Asia.

1. Al-Ghamdi, A. S., Analysis of time headways on urban roads: a case study from Riyadh. J. Transp. Syst. ASCE, 2001, 127, 289-294.
2. Arasan, V. and Koshi, R., Headway distribution of heterogeneous traffic on urban arterials. J. Inst. Eng., 2003, 84, 210-215.

3. Chandra, S. and Kumar, R., Headway modeling under mixed traffic on urban roads. Road Transp. Res., 2001, 10, 61-79.

4. Dey, P. P. and Chandra, S., Desired time gap and time headway in steady-state car-following on two-lane roads. J. Transp. Eng., 2009, 135, 687-693.

5. Katti, B. and Pathak, R., A study on headway distribution models for the urban road sections under mixed traffic conditions. Highway Research Bulletin 26, Indian Roads Congress. New Delhi, 1985, pp. 1-31.

6. Ramanayya, T., Highway capacity under mixed traffic conditions. Traf. Eng. Control, 1988, 29, 284-287.

7. Rossi, R., Gastaldi, M. and Pascucci, F., Gamma-GQM time headway model: endogenous effects in rural two-lane two-way roads. Proc. -Soc. Behav. Sci., 2014, 111, 859-868.

8. Panichpapiboon, S., Time-headway distributions on an expressway: case of Bangkok. J. Transp. Eng. ASCE, 2015, 141, 1-8.

9. Yun, M. and Huang, L., Distribution characteristics of headway at weaving section of signalized intersection upstream. International Conference on Transportation Engineering, ASCE, 2015, 13461353.

10. Tan, J., Li, L., Li, Z. and Zhang, Y., Distribution models for start-up lost time and effective departure flow rate. Transp. Res. Part A, 2013, 51, 1-11.

11. Woo, H. T. and Hoel, L. A., Toll plaza capacity and level of service. Transp. Res. Rec.: J. Transp. Res. Board, 1991, 1320, 119-127.

12. Zarrillo, M. L. and Radwan, A. E., Methodology SHAKER and the capacity analysis of five toll plazas. J. Transp. Eng. ASCE, 2009, 135, 83-93.

13. Osborne, R. P., Implementing Toll Plaza Analysis into Freeplan, University of Florida, USA, 2012.

14. Liu, X., Yun, M. and Yang, X., Lane capacity estimation and level of service evaluation for freeway toll plazas based on transaction data. In 97th TRB Annual Meeting, Transportation Research Board, Washington, DC, USA, 2018, pp. 1-8.

15. Navandar, Y. V., Dhamaniya, A., Patel, D. A. and Chandra, S., Traffic flow analysis at manual tollbooth operation under mixed traffic conditions. J. Transp. Eng. Part A, ASCE, 2019, 145(6), $1-17$.

16. Jang, J., Park, C., Kim, B. and Choi, N., Modeling of time headway distribution on suburban arterial: case study from South Korea. Procedia-Soc. Behav. Sci., 2011, 16, 240-247. 
17. Zhang, G., Wang, Y., Wei, H. and Chen, Y., Examining headway distribution models with urban freeway loop event data. Transp. Res. Rec.: J. Transp. Res. Board, 2007, 1999, 141-149.

18. Chen, D. and Xiaozheng, H., An evaluation method for toll policy in transportation. International Conference on E-Business and E-Government, 2010, pp. 618-621.

19. Riccardo, R. and Massimiliano, G., An empirical analysis of vehicle time headways on rural two-lane two-way roads. Procedia. - Soc. Behav. Sci., 2012, 54, 865-874.

20. Dubey, S. K., Ponnu, B. and Arkatkar, S. S., Time gap modeling using mixture distributions under mixed traffic conditions. J. Transp. Syst. Eng. Inf. Technol., 2013, 12, 72-84.

21. Zhang, G. and Wang, Y., A Gaussian kernel-based approach for modeling vehicle headway distributions. Transp. Sci., 2013, 48, 206-216.

22. Dong, S., Wang, H., Hurwitz, D., Zhang, G. and Shi, J., Nonparametric modeling of vehicle-type-specific headway distribution in freeway work zones. J. Transp. Eng. ASCE, 2015, 141, 05015004.

23. Kumar, P., Arkatkar, S. and Joshi, G., Examining traffic flow parameters at merging section on high-speed urban roads in India. Curr. Sci., 2019, 117, 94-103.

24. Mondal, S. and Gupta, A., Assessment of vehicles headway during queue dissipation at signal-controlled intersection under mixed traffic. Curr. Sci., 2019, 116, 437-444.

25. Mathwave, EasyFit Software Manual, Version 5.4, 2010; https://www.mathwave.com/downloads.

26. Mahapatra, G. and Maurya, A. K., Dynamic parameters of vehicles under heterogeneous traffic stream with non-lane discipline: an experimental study. J. Traff. Transp. Eng. (English Ed.), 2018, 5, 386-405.

27. Espinoza Mondragón, J., Jiménez García, J. A., Medina Flores, J. M., Vázquez López, J. A. and Téllez Vázquez, S., Experiments simulation and design to set traffic lights' operation rules. Transp. Policy, 2018, 67, 2-12.
28. Khraibani, R., de Palma, A., Picard, N. and Kaysi, I., A new evaluation and decision making framework investigating the elimination-by-aspects model in the context of transportation projects' investment choices. Transp. Policy, 2016, 48, 67-81.

29. Ghasemi, S. H., Jalayer, M., Mahdi, P.-R., Nowak, A. S. and Zhou, H., State-of-the-art model to evaluate space headway based on reliability analysis. J. Transp. Eng. ASCE, 2016, 142, 1-9.

30. Chandra, R. and Mujumdar, P. P., Quantification of uncertainty in spatial return levels of urban precipitation extremes. J. Hydrol. Eng. ASCE, 2017, 23, 2-14.

31. Chepuri, A., Ramakrishnan, J., Arkatkar, S., Joshi, G. and Pulugurtha, S. S., Examining travel time reliability-based performance indicators for bus routes using GPS-based bus trajectory data in India. J. Transp. Eng. Part A, ASCE, 2018, 144(5), 04018012.

32. Bari, C., Navandar, Y. and Dhamaniya, A., Service time variation analysis at manually operated toll plazas under mixed traffic conditions in India. J. East Asia Soc. Transp. Stud., 2019, 13, $331-350$.

33. Indian Roads Congress, Manual of Specifications and Standards for Four Laning of Highways through Public Private Partnership, IRC SP: 84, 2014.

34. Teena, N. V., Sanil Kumar, V., Sudheesh, K. and Sajeev, R., Statistical analysis on extreme wave height. Nat. Hazards, 2012, 64, 223-236.

35. Friederichs, P. and Thorarinsdottir, T. L., Forecast verification for extreme value distributions with an application to probabilistic peak wind prediction. Environmetrics, 2012, 23, 579-594.

Received 14 May 2020; revised accepted 31 March 2021

doi: $10.18520 / \mathrm{cs} / \mathrm{v} 121 / \mathrm{i} 1 / 148-160$ 